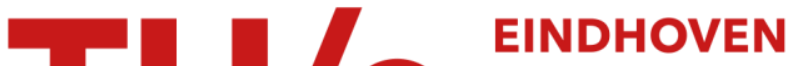

\section{An analysis of the highly linear transfer characteristics of dual- buck converters}

Citation for published version (APA):

Schellekens, J. M., Huisman, H., Duarte, J. L., Hendrix, M. A. M., \& Lomonova, E. A. (2018). An analysis of the highly linear transfer characteristics of dual-buck converters. IEEE Transactions on Industrial Electronics, 65(6), 4681-4690. https://doi.org/10.1109/TIE.2017.2772175

DOI:

10.1109/TIE.2017.2772175

Document status and date:

Published: 01/06/2018

Document Version:

Accepted manuscript including changes made at the peer-review stage

Please check the document version of this publication:

- A submitted manuscript is the version of the article upon submission and before peer-review. There can be important differences between the submitted version and the official published version of record. People interested in the research are advised to contact the author for the final version of the publication, or visit the $\mathrm{DOI}$ to the publisher's website.

- The final author version and the galley proof are versions of the publication after peer review.

- The final published version features the final layout of the paper including the volume, issue and page numbers.

Link to publication

\section{General rights}

Copyright and moral rights for the publications made accessible in the public portal are retained by the authors and/or other copyright owners and it is a condition of accessing publications that users recognise and abide by the legal requirements associated with these rights.

- Users may download and print one copy of any publication from the public portal for the purpose of private study or research.

- You may not further distribute the material or use it for any profit-making activity or commercial gain

- You may freely distribute the URL identifying the publication in the public portal.

If the publication is distributed under the terms of Article $25 \mathrm{fa}$ of the Dutch Copyright Act, indicated by the "Taverne" license above, please follow below link for the End User Agreement:

www.tue.nl/taverne

Take down policy

If you believe that this document breaches copyright please contact us at:

openaccess@tue.nl

providing details and we will investigate your claim. 


\title{
An analysis of the highly linear transfer characteristics of dual-buck converters
}

\author{
Jan M. Schellekens, Henk Huisman, Senior Member, IEEE, Jorge L. Duarte, Member, IEEE, \\ Marcel A. M. Hendrix Member, IEEE, and Elena A. Lomonova, Senior Member, IEEEof Electrical \\ Engineering, Eindhoven University of Technology, Eindhoven 5612AZ, The Netherlands, \\ (email:j.m.schellekens@tue.nl).
}

\begin{abstract}
Practical switching devices have finite turnon and turn-off times. To avoid short circuit, a blanking time is added between turn-off and turn-on of the complementary working switches in a switching-leg. The blanking time, also referred to as dead-time, is one of the dominant sources of output current and voltage distortion in pulsewidth modulated power amplifiers.

Extensive studies exist on elimination, minimization, and compensation of the effect. Most techniques achieve a reduction of the distortion but are not capable of completely removing it. The dual-buck converter does not suffer from blanking-time-related distortion. However, blanking time is not the only source of switching-leg-induced distortion.

This paper focuses on the effects of semiconductor device parameters on the output quality of the dual-buck converter. It is shown that, ideally, the forward voltages of the diodes and switches have no effect on the output quality. Measurements on a prototype, industrial power stack based, dual-buck converter show a 100 times improvement of the open-loop spurious free dynamic range when compared to conventional pulse width modulated converters.
\end{abstract}

\section{INTRODUCTION}

B LANKING time is one of the most dominant sources of output voltage distortion for pulse width modulated (PWM) amplifiers based on the conventional switching-leg depicted in Fig. 1a. High-precision applications, like shortstroke linear mechanical actuators for wafer steppers, gradient coils for magnetic resonance imaging (MRI) systems, and studio-quality audio, that require better than $80 \mathrm{~dB}$ dynamic range, suffer especially from the blanking-time effect.

Feedback is normally applied in converters to reject disturbances. The impact of blanking time can indeed be reduced by feedback. However, due to the extremely fast change of the output voltage as a function of current and modulation index, as pointed out in [1] and further detailed in [2], it is not practical to eliminate the distortion due to blanking time with feedback only. However, improvements can be gained by using interleaving or multilevel techniques, as suggested in many papers including [3]-[5], to allow higher bandwidth and better disturbance rejection of the closed loop converter system compared to conventional 2-level PWM converters.

Extensive studies have been done on the elimination [6], minimization [7], and compensation [8]-[10] of blanking time in PWM converters. Most of these techniques rely on the detection of the polarity of the sampled inductor current of the converter and neglect the switching transients.
In [11] a compensation scheme is suggested that includes the inductor current ripple, and in [12] a more advanced model of the switch-node commutation was added. All techniques mentioned above achieve a reduction of the distortion. However, due to errors in the detection of current polarity and discontinuous conduction mode (DCM) during zero crossings, they are not capable of completely removing it.

Different modulation techniques have been suggested that alleviate blanking time effects [6], [7], [9], [13], however, these methods also rely on the polarity of the current and do not completely remove the impact of blanking time. In [14]-[17] the amount of blanking time is reduced (adaptively or actively) to the absolute minimum at the cost of increased losses.

The resonant pole inverter (RPI) discussed in [18]-[20] features zero-voltage switching (ZVS), and therefore does not suffer from blanking-time-related distortion. However, to achieve ZVS the RPI requires considerable circulating current. Nearly zero-voltage turn-off in the RPI is achieved by reducing the slew rate of the switch-node voltage by adding capacitance in parallel with the switches. The resulting lower slew rate during commutation leads to reduced electromagnetic interference (EMI). As a result a well designed RPI does not require additional EMI filtering, as is normally required in conventional PWM converters. The slew rate of the switching transients, however, is strongly dependent on the operating point of the converter and adds significant distortion.

In practice, the output-voltage quality of RPIs is better than that of conventional PWM converters, but not sufficient for many high-precision applications. Furthermore, the hysteresis control scheme of RPIs results in variable switching frequency and is hard to implement digitally. The RPI is, therefore, rarely used in industry.

In [21] a circuit consisting of two parallel-connected down converters, one for positive current and one for negative current, is presented as a high-performance converter that does not suffer from blanking-time-related distortion and is robust for shoot-through, i.e. unintentional turn-on of two semiconductor switches simultaneously. It was shown that with proper control, low distortion can be achieved, even when nonlinear loads are connected. Later, in [22], the circuit was introduced as a buck derived PWM power stage with reduced shoot-through current, allowing zero blanking-time operation, and was baptized opposed current converter (OCC). Operation of multiple parallel OCC stages was proposed, and it was shown that the topology is suitable for studio-quality 


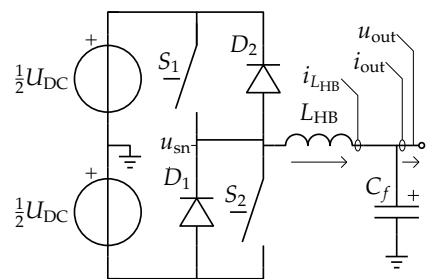

(a)

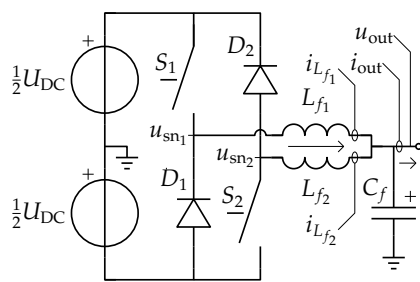

(b)
Fig. 1. Schematic diagrams of (a) the conventional HB switching-leg, and (b) the DB switching-leg, both with output filters.

audio reproduction and high-precision positioning systems. In [23] hysteresis current control was proposed for the OCC, and it was renamed to dual-buck (DB) converter. Later, the same topology started to appear as split-phase dual-buck [24]. In the remainder of this paper the parallel-connected down converters, as shown in Fig. 1b, will be referred to as DB.

A disadvantage of the DB is that it requires 2 filter inductors and thus more volume compared to conventional PWM converters. Studies show that the total volume can be reduced by coupling of the inductors [25], by changing the configuration of the inductors [26], or by altering the configuration of the switching legs [27]. However, the total inductor volume will remain larger than conventional PWM converters by at least factor 1.5 .

The dual-buck converter does not require blanking time and, therefore, does not suffer from zero-crossing distortion due to blanking time. However, other phenomena, like nonideal switches, diodes and inductors, contribute to harmonic distortion as well. In conventional converters these effects are, in practice, often neglected, since their contribution is relatively small compared to the effect of blanking time, or simply because high accuracy is not an issue. In [28], and more extensively in [29], different sources of distortion in switching amplifiers are discussed. These studies focus on the effects of bipolar junction transistor switches. However, many of the ideas can be applied to other types of semiconductor switches too. In [30] a detailed analysis of distortion introduced by power metal oxide semiconductor field effect transistors (MOSFETs) in class-D audio amplifiers was made. These studies concentrate on the conventional switching leg, and do not apply to the dual buck converter. In [31] a simulation study shows that the DB is superior to the conventional half-bridge (HB) converter. The total harmonic distortion (THD) of the $\mathrm{DB}$ is shown to be $12 \mathrm{~dB}$ better compared to a HB stage with extremely small blanking-time. However, a detailed paper with an analysis of why the DB converter is so much better than the conventional HB is still missing.

This paper presents the main results of [25] and focuses on modulation artifacts, the effects of forward voltage and resistance of the semiconductors, and the series resistances of filter components on the output quality of a DB. It is shown that the average behavior of the DB can be made linear by proper design. Finally, the theory is verified with measurements on a prototype based on an industrial inverter.

\section{THE PULSE-WIDTH MODULATED DUAL-BUCK CONVERTER}

Fig. 1b depicts a practical implementation of the DB switching leg with filtering of the PWM voltage waveforms. The switching components that supply positive current to the load are indicated with subscript 1 , and the switching components that supply negative current are indicated with subscript 2. Furthermore, the filter inductors have equal values, $L_{f}$, and all voltages are referenced to the center terminal of the symmetrical DC supply.

The periodic average switch-node voltages $\left\langle u_{\mathrm{sn}_{x}}\right\rangle$ can be determined by applying the state-space averaging method as presented in [32]. When disregarding losses, assuming continuous conduction mode (CCM) operation, and instantaneous switching they become

$$
\left\langle u_{\mathrm{sn}_{x}}\right\rangle=\frac{1}{2} U_{\mathrm{DC}} m_{x}
$$

where the subscript $x$ indicates the corresponding switching leg, $m_{x}$ is the modulation index, which is limited to the interval $[-1 \ldots 1]$, and $U_{\mathrm{DC}}$ is the DC supply voltage of the DB switching leg. From (1) it can be seen that ideally a linear relation exists between the switch-node voltages and modulation indices when both legs of the DB are operated in CCM. For discontinuous conduction mode (DCM) that relation becomes nonlinear, as shown in [1], and should thus be avoided for high quality output.

Both legs are effectively operated in parallel and can be analyzed separately when assuming $u_{C_{f}}$ constant over one switching cycle $\left(T_{\mathrm{sw}}\right)$. This is approximately valid because in practice the series resistance of $C_{f}$ can be neglected, and $C_{f}$ is chosen large enough that the output voltage is smooth. When assuming $u_{C_{f}}$ constant and CCM operation, the steady-state inductor current ripple amplitude $\left(\Delta i_{L_{f}}\right)$ becomes

$$
\begin{aligned}
\Delta i_{L_{f_{x}}} & =\hat{\Delta} i_{L_{f}}\left(1-m_{x}{ }^{2}\right) \quad \text { with } \\
\hat{\Delta} i_{L_{f}} & =\frac{U_{\mathrm{DC}}}{8 L_{f}} T_{\mathrm{sw}}
\end{aligned}
$$

where $T_{\mathrm{sw}}$ is the switching cycle time of the PWM, and $L_{f}$ is the filter inductance.

\section{A. Decoupling through variable transformation}

To ensure CCM under all output conditions, a bias current is required to flow from the positive-current leg to the negativecurrent leg. Therefore, it is convenient to separate this bias voltage and current from the voltage and current that drives the output. Doing so leads to decoupled control of the output power and the current necessary to maintain CCM. Fig. 2 depicts the inductor and output currents, and the output voltage on the left, where the band around the inductor current represents the current ripple of (2a). Note that $i_{L_{f_{1}}}$ is always positive, and $i_{L_{f_{2}}}$ is negative. The right graph shows the decoupled quantities where the voltages are indicated as cycle averages. The following transformation can be used for the decoupling:

$$
\begin{aligned}
u_{\mathrm{avg}} & =\frac{1}{2}\left(u_{\mathrm{sn}_{1}}+u_{\mathrm{sn}_{2}}\right) \\
u_{\mathrm{bias}} & =u_{\mathrm{sn}_{1}}-u_{\mathrm{sn}_{2}}
\end{aligned}
$$




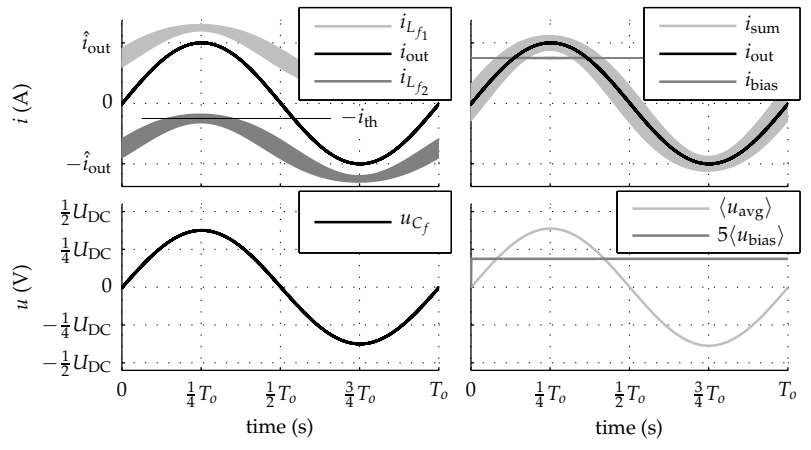

Fig. 2. Output current and voltage waveforms with constant bias current.

where $u_{\text {avg }}$ is the average switch-node voltage of the $\mathrm{P}$ - and $\mathrm{N}$-cell, and $u_{\text {bias }}$ is the difference between the switch-node voltages which drives the bias current. A similar transformation for the currents is given by

$$
\begin{aligned}
& i_{\text {sum }}=i_{L_{f_{1}}}+i_{L_{f_{2}}} \\
& i_{\text {bias }}=\frac{1}{2}\left(i_{L_{f_{1}}}-i_{L_{f_{2}}}\right)
\end{aligned}
$$

where $i_{\text {sum }}$ is the combined current of the output filter inductors, and $i_{\text {bias }}$ is the current required to prevent DCM, and consequently a nonlinear relation between control input and output.

DCM can be prevented by ensuring that the moving average inductor currents are larger than the corresponding inductor current ripple amplitudes, i.e. $\left\langle i_{L_{f_{1}}}\right\rangle \geq \Delta i_{L_{f_{1}}}$ and $\left\langle i_{L_{f_{2}}}\right\rangle \leq$ $\Delta i_{L_{f_{2}}}$, basically meaning that

$$
\left\langle i_{\text {bias }}\right\rangle \geq \frac{1}{2}\left|\left\langle i_{\text {sum }}\right\rangle\right|+i_{\text {th }}
$$

with

$$
i_{\mathrm{th}} \geq \begin{cases}\Delta i_{L_{f_{2}}}, & \left\langle i_{\text {sum }}\right\rangle \geq 0 \\ \Delta i_{L_{f_{1}}}, & \left\langle i_{\text {sum }}\right\rangle<0 .\end{cases}
$$

The bias current can be kept constant and equal to the maximum expected $i_{\text {sum }}$ plus the offset current $i_{\text {th }}$, as shown in Fig. 2, or modulated as

$$
\left\langle i_{\text {bias }}\right\rangle=\frac{1}{2}\left|\left\langle i_{\text {sum }}\right\rangle\right|+i_{\text {th }}
$$

as depicted in Fig. 3 for sinusoidal output current. Notice that modulated bias current requires a step change of the $u_{\text {bias }}$ in the zero crossings of $i_{\text {sum }}$ because of the absolute operator in (7). Because ideally only an inductor is connected between the two DB legs, some means of current control is required for $i_{\text {bias. }}$.

A similar transformation can be used for the controlling inputs, leading to

$$
\begin{aligned}
& m_{\text {avg }}=\frac{1}{2}\left(m_{1}+m_{2}\right) \\
& m_{\text {bias }}=m_{1}-m_{2}
\end{aligned}
$$

where $m_{\mathrm{avg}}$ is the controlling modulation index for $\left\langle u_{\mathrm{avg}}\right\rangle$, and $m_{\text {bias }}$ for $\left\langle u_{\text {bias }}\right\rangle$. Finally, by combining (1) and (8) it follows that

$$
\begin{aligned}
\left\langle u_{\mathrm{avg}}\right\rangle & =\frac{1}{2} U_{\mathrm{DC}} m_{\mathrm{avg}} \\
\left\langle u_{\mathrm{bias}}\right\rangle & =\frac{1}{2} U_{\mathrm{DC}} m_{\mathrm{bias}}
\end{aligned}
$$

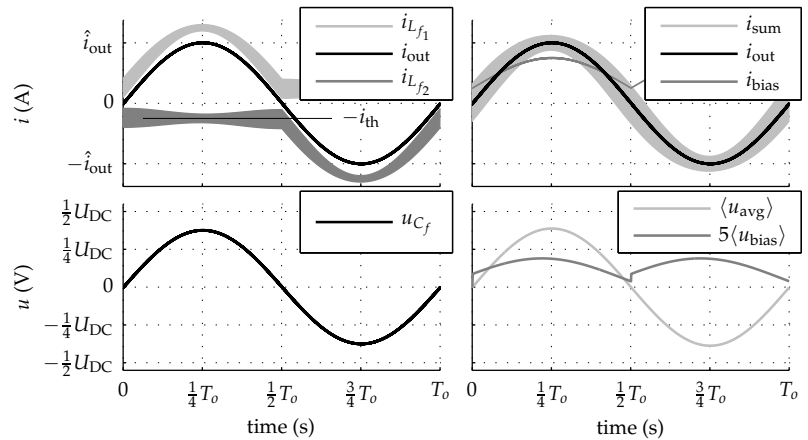

Fig. 3. Output current and voltage waveforms with modulated bias current.

which is valid when assuming no losses, CCM and instantaneous switching of the semiconductors.

It should be noted that the output voltage range of the DB converter is limited by the amount of bias voltage that is required, since the absolute value of the modulation indices $\left(m_{x}\right)$ cannot exceed one, which leads to the following boundary condition

$$
\left|m_{\text {avg }}\right|+\frac{1}{2} m_{\text {bias }} \leq 1 .
$$

The inductances $L_{f}$ can also be expressed in terms of $L_{\text {sum }}$ and $L_{\text {bias }}$, which for uncoupled inductors equals

$$
\begin{aligned}
& L_{\text {sum }}=\frac{1}{2} L_{f} \\
& L_{\text {bias }}=2 L_{f} .
\end{aligned}
$$

For coupled inductors it is possible to choose the ratio between the sum and bias inductance with the coupling coefficient as discussed in [25].

The variable transformation presented in this section decouples the bias and output related voltages and currents, and is henceforth applied to the DB converter.

\section{B. PWM generation}

Because the rate of current change is limited by the inductors that couple the switching-leg voltages, the gating signals for a DB switching leg do not need to be complementary and may even overlap. This adds an additional degree of freedom to the PWM patterns of the DB switching leg. In the ideal case only two possible PWM methods make sense; to switch both legs center-aligned (non-interleaved) or time shifted by $\frac{1}{2} T_{\mathrm{sw}}$ (interleaved).

Fig. 4 and Fig. 5 depict the switching waveforms for the DB switching leg for non-interleaved and interleaved switchnode voltage $\left(u_{\mathrm{sn}}\right)$, respectively, with and without variable transformation. Losses are included in the simulation, resulting in $u_{\text {bias }}=0.04 U_{\mathrm{DC}}$, which leads to a visible difference in pulse width between $u_{\mathrm{sn}_{1}}$ and $u_{\mathrm{sn}_{2}}$. Fig. 4 and Fig. 5 also illustrate the deviation due to the voltage drop across the resistive element of the semiconductors, of the switch-node voltages from a perfect square wave.

The bias voltage is especially visible for non-interleaved $u_{\mathrm{sn}}$ in Fig. 4 where it appears in the form of narrow voltage peaks. In Fig. 5 the bias voltage appears as slightly wider positive $u_{\text {bias }}$ pulses compared to the negative $u_{\text {bias }}$ pulses. 

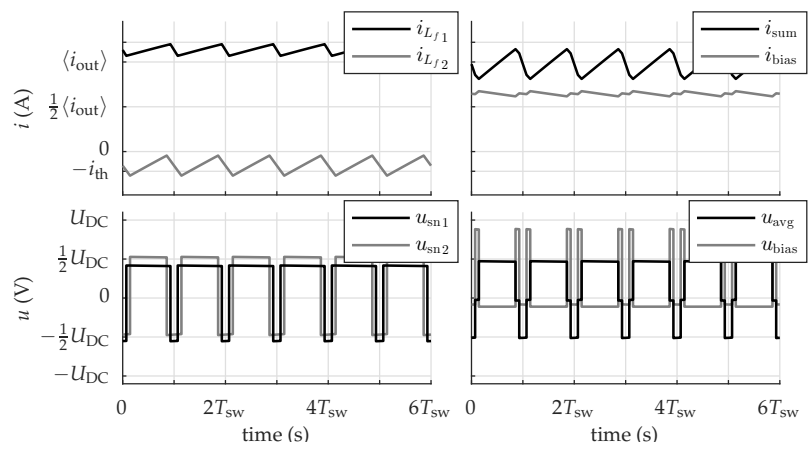

Fig. 4. Switching waveforms of the DB switching leg, for non-interleaved $u_{\mathrm{sn}}$.

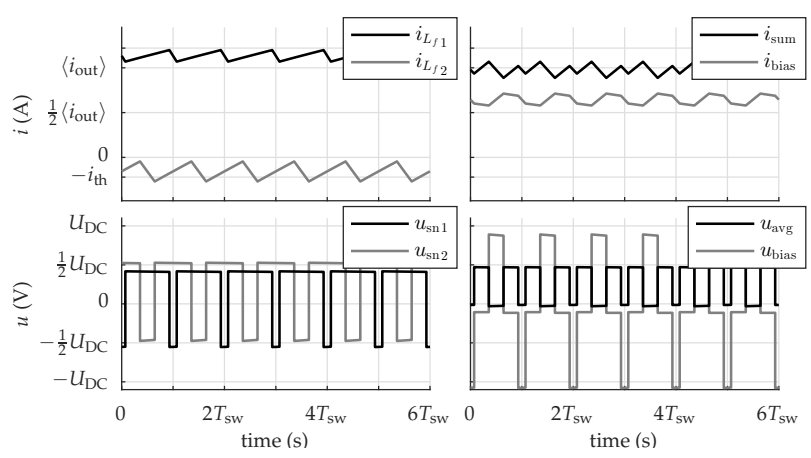

Fig. 5. Switching waveforms of the DB switching leg, for interleaved $u_{\mathrm{sn}}$.

When $\left\langle u_{\text {bias }}\right\rangle=0$, in case of non-interleaved voltages, as depicted in Fig. $4, i_{\text {bias }}$ and $u_{\text {bias }}$ have no ripple, and $i_{\text {sum }}$ and $u_{\text {avg }}$ have ripple with frequency equal to the switching frequency $\left(f_{\mathrm{sw}}\right)$. For interleaved voltages, as in Fig. 5, the frequency of the ripple of $i_{\text {sum }}$ and $u_{\text {avg }}$ doubles, and the ripple amplitudes halve. However, a nonzero ripple with fundamental frequency equal to $f_{\mathrm{sw}}$ appears on $i_{\text {bias }}$ and $u_{\text {bias. }}$. Consequently, there is a trade-off between the effective frequency and the amplitude of the ripple of $u_{\text {avg }}$ and $i_{\text {sum }}$ on one hand, and the ripple of $u_{\text {bias }}$ and $i_{\text {bias }}$ on the other hand. Also notice that for non-interleaved $u_{\mathrm{sn}}$ the fundamental switching frequency component appears in the bias voltage and current. For interleaved $u_{\mathrm{sn}}$ the fundamental switching frequency component appears in $u_{\text {avg }}$ and $i_{\text {sum }}$ when $u_{\text {bias }}>0$, making interleaving less effective for the DB.

\section{THE IMPACT OF COMPONENT PARAMETERS ON LINEARITY OF THE DUAL BUCK}

In this section the effects of the forward voltages and series resistances of the diodes and switches $\left(V_{f}, V_{\mathrm{on}}, R_{f}, R_{\mathrm{on}}\right)$, and the series resistance $\left(R_{L_{f}}, R_{C_{f}}\right)$ of the filter components $\left(L_{f}\right.$, $C_{f}$ ), are investigated based on the model presented in Fig. 6.

Since current can flow only in one direction through the switch in each of the split legs, antiparallel diodes are not strictly required. This means that the model depicted in Fig. 6 can be applied to investigate the effects of the voltage drops across the components for both unipolar current switches, such as IGBTs, and bipolar-current switches, such as MOSFETs.

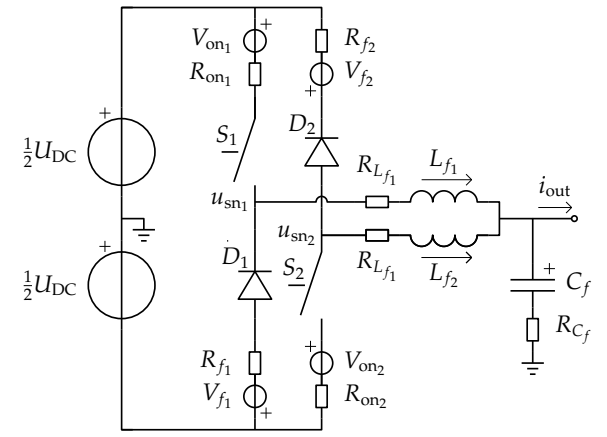

Fig. 6. DB switching leg with parasitic components.

\section{A. Periodically averaged model}

The state-space averaging method is used to derive an average model that describes the impact of the component parameters shown in Fig. 6. The following, not yet linearized, averaged state-space representation can be obtained

$$
\langle\dot{\mathbf{x}}\rangle \approx\langle\mathbf{A}(\mathbf{u})\rangle\langle\mathbf{x}\rangle+\langle\mathbf{b}(\mathbf{u})\rangle .
$$

where \langle\rangle denotes a switching cycle average value.

When the state vector and input vector are chosen to be

$$
\begin{aligned}
& \mathbf{x}=\left(\begin{array}{lll}
i_{L_{f_{1}}} & i_{L_{f_{2}}} & u_{C_{f}}
\end{array}\right)^{\top}, \text { and } \\
& \mathbf{u}=\left(\begin{array}{lll}
m_{1} & m_{2} & i_{\text {out }}
\end{array}\right)^{\top}
\end{aligned}
$$

respectively, the state matrix becomes

$$
\langle\mathbf{A}(\mathbf{u})\rangle=\left(\begin{array}{ccc}
-\frac{R_{1}^{\prime}\left(m_{1}\right)}{L_{f_{1}}} & -\frac{R_{C_{f}}}{L_{f_{1}}} & -\frac{1}{L_{f_{1}}} \\
-\frac{R_{C_{f}}}{L_{f_{2}}} & -\frac{R_{2}^{\prime}\left(m_{2}\right)}{L_{f_{2}}} & -\frac{1}{L_{f_{2}}} \\
\frac{1}{C_{f}} & \frac{1}{C_{f}} & 0
\end{array}\right)
$$

with

$$
\begin{aligned}
& R_{1}^{\prime}\left(m_{1}\right)=R_{L_{f_{1}}}+R_{C_{f}}+R_{\mathrm{on}_{1}}+R_{\mathrm{on}_{1}}-\left(R_{f_{1}}-R_{\mathrm{on}_{1}}\right) m_{1} \\
& R_{2}^{\prime}\left(m_{2}\right)=R_{L_{f_{2}}}+R_{C_{f}}+R_{\mathrm{on}_{2}}+R_{\mathrm{on}_{2}}+\left(R_{f_{2}}-R_{\mathrm{on}_{2}}\right) m_{2} .
\end{aligned}
$$

Finally, the averaged input vector is determined to be

$$
\langle\mathbf{b}(\mathbf{u})\rangle=\left(\begin{array}{c}
\frac{-\frac{1}{2}\left(V_{f_{1}}+V_{\mathrm{on}_{1}}\right)+\frac{1}{2}\left(U_{\mathrm{DC}}+V_{f_{1}}-V_{\mathrm{on}_{1}}\right) m_{1}-R_{C_{f}} i_{\mathrm{out}}}{L_{f_{1}}} \\
\frac{\frac{1}{2}\left(V_{f_{2}}+V_{\mathrm{on}_{2}}\right)+\frac{1}{2}\left(U_{\mathrm{DC}}+V_{f_{2}}-V_{\mathrm{on}_{2}}\right) m_{2}+R_{C_{f}} i_{\mathrm{out}}}{L_{f_{2}}} \\
-\frac{i_{\mathrm{out}}}{C_{f}}
\end{array}\right) .
$$

It should be noted that the averaged result is an approximation. However, when $\langle\mathbf{A}\rangle$ is time-invariant, i.e. when it is not a function of $\mathbf{u}(t)$, the averaged model given in (12) exactly describes the moving-average behavior of the DB model presented in Fig. 6. For the DB the state matrix (15) becomes time-invariant when $R_{f_{1}}=R_{\mathrm{on}_{1}}$ and $R_{f_{2}}=R_{\mathrm{on}_{2}}$, i.e. when the resistances of the diodes and switched are matched. Thus, when the resistances of the switches and diodes of each leg are matched, the cycle averaged behavior of the DB converter leg is linear and can be modeled using state-space averaging without error. A full derivation of the average model can be found in [25]. 


\section{B. Steady-state solution}

When assuming steady-state, i.e. $\langle\dot{\mathbf{x}}\rangle=0, I_{\text {out }}$ equals $\left\langle I_{L_{f_{1}}}\right\rangle+\left\langle I_{L_{f_{2}}}\right\rangle$, where the capitals represent steady-state values as the bottom rows of (15) and (16) are the same, and contain no useful information. The remaining two steadystate equations can be expressed in terms of desired voltages by application of the transformations given in (4), (8), and by rewriting the modulation indices in desired voltages similarly as in (8).

The two resulting expressions are straightforward to solve but the results are lengthy and not insightful. However, when assuming equal forward voltages and on-resistances for the diodes and switches, i.e. $V_{f}=V_{f_{x}}, R_{f}=R_{f_{x}}, V_{\mathrm{on}}=V_{\mathrm{on}_{x}}$, and $R_{\mathrm{on}}=R_{\mathrm{on}_{x}}$, with $x=\{1,2\}$, the expressions become

$$
\begin{array}{r}
U_{\mathrm{avg}}^{*} \approx \frac{U_{\mathrm{DC}}}{2\left(c+a I_{L_{f_{1}}}\right)\left(c-a I_{L_{f_{2}}}\right)} \times\left(k I_{L_{f_{1}}}+l I_{L_{f_{2}}}\right. \\
\left.-a\left(R_{L_{f_{1}}}-R_{L_{f_{2}}}\right) I_{L_{f_{1}}} I_{L_{f_{2}}}+c 2 U_{C_{f}}\right)
\end{array}
$$

and

$$
\begin{aligned}
U_{\text {bias }}^{*} & \approx \frac{U_{\mathrm{DC}}}{\left(c+a I_{L_{f_{1}}}\right)\left(c-a I_{L_{f_{2}}}\right)} \times\left(m I_{L_{f_{1}}}-n I_{L_{f_{2}}}\right. \\
& \left.-a\left(R_{L_{f_{1}}}+R_{L_{f_{2}}}+R_{f}+R_{\mathrm{on}}\right) I_{L_{f_{1}}} I_{L_{f_{2}}}+d\right)
\end{aligned}
$$

respectively, where

$$
\begin{aligned}
a & =R_{f}-R_{\mathrm{on}} \\
b & =\frac{1}{2}\left(R_{f}+R_{\mathrm{on}}\right) \\
c & =U_{\mathrm{DC}}+V_{f}-V_{\mathrm{on}} \\
d & =\left(V_{f}+V_{\mathrm{on}}\right) U_{\mathrm{DC}}+V_{f}^{2}-V_{\mathrm{on}}^{2}
\end{aligned}
$$

and

$$
\begin{aligned}
k & =b U_{\mathrm{DC}}+a U_{C_{f}}+c R_{L_{f_{1}}}+R_{\mathrm{on}} V_{f}-R_{f} V_{\mathrm{on}} \\
l & =b U_{\mathrm{DC}}-a U_{C_{f}}+c R_{L_{f_{2}}}+R_{\mathrm{on}} V_{f}-R_{f} V_{\mathrm{on}} \\
m & =b U_{\mathrm{DC}}-a U_{C_{f}}+c R_{L_{f_{1}}}+R_{f} V_{f}-R_{\mathrm{on}} V_{\mathrm{on}} \\
n & =b U_{\mathrm{DC}}+a U_{C_{f}}+c R_{L_{f_{2}}}+R_{f} V_{f}-R_{\mathrm{on}} V_{\mathrm{on}} .
\end{aligned}
$$

Notice that, due to the steady-state assumption, inductances $L_{f_{1}}$ and $L_{f_{2}}$, capacitance $C_{f}$, and resistance $R_{C_{f}}$ are eliminated from (17) and (18), and that it is a nonlinear approximation because the result is based on a time-variant state matrix.

When $R^{\prime}=R_{f}=R_{\mathrm{on}}$, (17) and (18) respectively simplify to

$$
\begin{aligned}
U_{\mathrm{avg}}^{*}=\frac{U_{\mathrm{DC}}}{U_{\mathrm{DC}}+V_{f}-V_{\mathrm{on}}} & \left(U_{C_{f}}\right. \\
+\left(\frac { 1 } { 4 } \left(R_{L_{f_{1}}}+\right.\right. & \left.\left.R_{L_{f_{2}}}\right)+\frac{1}{2} R^{\prime}\right) I_{\text {sum }} \\
& \left.+\frac{1}{2}\left(R_{L_{f_{1}}}-R_{L_{f_{2}}}\right) I_{\mathrm{bias}}\right)
\end{aligned}
$$

and

$$
\begin{aligned}
& U_{\text {bias }}^{*}=\frac{U_{\mathrm{DC}}}{U_{\mathrm{DC}}+V_{f}-V_{\mathrm{on}}}\left(V_{f}+V_{\mathrm{on}}\right. \\
&+\left(R_{L_{f_{1}}}+R_{L_{f_{2}}}+2 R^{\prime}\right) I_{\mathrm{bias}}+ \\
&\left.\frac{1}{2}\left(R_{L_{f_{1}}}-R_{L_{f_{2}}}\right) I_{\mathrm{sum}}\right)
\end{aligned}
$$

where $I_{\text {sum }}$ and $I_{\text {bias }}$ are steady-state currents given by (4). Both (19) and (20) are linear. Furthermore, it can be seen that the forward voltages of the semiconductor switches and diodes only lead to an additional static gain error and, therefore, do not result in harmonic distortion like in conventional switching legs, as was already pointed out in [28]. The forward voltage loss, however, needs to be compensated with the bias voltage reference (20) and, therefore, imposes a restriction of the maximum output voltage range of the DB switching leg, as pointed out in (10). There is, however, cross coupling between $U_{\text {avg }}^{*}$ and $I_{\text {bias }}$, and $U_{\text {bias }}^{*}$ and $I_{\text {sum }}$.

By choosing the same series resistances for both inductors, that is $R_{L_{f}}=R_{L_{f_{1}}}=R_{L_{f_{2}}}$, the cross-coupling can be removed, resulting in

$$
U_{\mathrm{avg}}^{*}=\frac{U_{\mathrm{DC}}}{U_{\mathrm{DC}}+V_{f}-V_{\mathrm{on}}}\left(U_{C_{f}}+\frac{1}{2}\left(R_{L_{f}}+R^{\prime}\right) I_{\mathrm{sum}}\right)
$$

and

$$
\begin{aligned}
U_{\text {bias }}^{*}=\frac{U_{\mathrm{DC}}}{U_{\mathrm{DC}}+V_{f}-V_{\mathrm{on}}}\left(V_{f}\right. & +V_{\mathrm{on}} \\
& \left.+2\left(R_{L_{f}}+R^{\prime}\right) I_{\mathrm{bias}}\right)
\end{aligned}
$$

respectively, which are both linear and have no cross coupling. Matching of the inductors to prevent cross coupling is especially important for modulated bias current, where $i_{\text {bias }}$ is modulated nonlinearly as given in (5). For constant bias current ideally only a static error is added to the output quantity due to cross coupling. Again, the forward voltage drops of the switches and diodes only lead to an additional static gain error and not to harmonic distortion.

In practice the series resistances of the switches and diodes are not equal. This can be compensated by matching the resistances of the switches and diodes. Adding an extra transistor, which is always on, in series with the diodes, and an additional diode in series with the transistors, results in a perfectly matched switching leg. However, losses will increase. Another symmetrization approach is to apply synchronous rectification by placing switches in parallel with the diodes in the DB leg. The synchronous rectification switches should be switched on when the diode would normally conduct.

Experiments with both matched circuits were done in [31] and showed that in practice synchronous rectification gives the best results for $\mathrm{GaN}$ and $\mathrm{SiC}$ switches. This is due to the fact that synchronous rectification results in lower effective $R_{\text {on }}$ and $R_{f}$, and consequently smaller difference between $R_{\text {on }}$ and $R_{f}$, compared to matching with series diodes and switches. Therefore it is important to keep variations between the parasitic resistances, and the filter inductances, static and varying due to e.g. temperature and saturation, small compared to the load impedance.

In practice the voltage drop of the diodes and switches depends nonlinearly on temperature and current. As such, the temperatures of the devices need to be matched as close as possible, which is challenging in reality due to the operatingpoint dependency of the semiconductor losses. Still, the results of this section can be used to calculate precise, operatingpoint-dependent, steady-state feed-forward references for the DB. 
TABLE I

CONDUCTION-RELATED PARAMETERS OF SWITCHES, DIODES AND INDUCTORS $\left(L_{f}\right)$ USED IN SIMULATIONS.

\begin{tabular}{lccccc}
\hline & $V_{\text {on }}(\mathrm{V})$ & $R_{\text {on }}(\mathrm{m} \Omega)$ & $V_{f}(\mathrm{~V})$ & $R_{f}(\mathrm{~m} \Omega)$ & $R_{L_{f}}(\mathrm{~m} \Omega)$ \\
\hline Matched & 1.7 & 40 & 1.2 & 40 & 50 \\
IGBT & 1.7 & 40 & 1.2 & 22 & 50 \\
MOSFET & 0 & 109 & 1.2 & 22 & 50 \\
\hline
\end{tabular}

\section{THE IMPACT OF COMPONENT PARAMETERS ON OUTPUT QUALITY OF THE DUAL BUCK}

The averaged model is verified using simulation results of the DB model depicted in Fig. 6, with a resistive load. The device parameters for the IGBT cases are determined from the datasheet of the Semikron SKM75GB123D IGBT, the same device as used in the experimental setup. For the MOSFET case, parameters are taken from a $1200 \mathrm{~V}$ silicon MOSFET scaled to the same current rating as the IGBT. The diode parameters for all three cases were taken from the integrated diode of the IGBT. However, for the matched case $R_{f}$ was chosen equal to the IGBT's on-resistance. The parameters used for simulation are summarized in Table I. The switching transients were assumed instantaneous for this analysis. More detailed results including voltage commutation can be found in [25].

Fig. 7 depicts the simulated open-loop output voltage spectrum, for asymmetrical regular-sampled PWM, as described in [33]. The output voltage $\left(u_{\text {out }}\right)$ that appears across the load resistance was sinusoidally modulated to half of the voltage range with a frequency of $21 \mathrm{~Hz}$. The resistive load was chosen such that the resulting current was also modulated to $50 \%$ of the output range, and $f_{\mathrm{sw}}$ was $16 \mathrm{kHz}$, which matches with the experiments presented later in this paper. The switches and diodes were modeled as indicated in Fig. 6.

Fig. 7 shows that the matched case does not suffer from harmonic distortion, as was predicted in the previous section. For constant bias, depicted in Fig. 7a, all harmonics of the IGBT case are smaller than $-117 \mathrm{~dB}$, and the third harmonic for the MOSFET case appears at approximately $-100 \mathrm{~dB}$, which is about a factor seven higher than the IGBT case. The harmonic distortion for modulated bias current, depicted in Fig. 7b, is much higher than for constant bias current. That occurs due the impact of unequal $R_{\text {on }}$ and $R_{f}$ of the semiconductor devices in (17). The effect of unequal $R_{\text {on }}$ and $R_{f}$ is in this particular case 2 orders of magnitude more severe for modulated $i_{\text {bias }}$ than for constant $i_{\text {bias }}$.It should be noted that the filter inductors were matched in this simulations study, as can be seen from Table I. In [25] the steady-state voltage error as function of modulation indices and output current is calculated for constant and modulated $i_{\text {bias }}$, and it is shown that, especially for small modulation indices, constant bias is superior over modulated bias. Constant bias current, however, comes at the cost of significant additional losses.

\section{EXPERIMENTAL RESULTS}

A prototype converter was built that can be configured as a full-bridge equivalent $\mathrm{DB}$ or a conventional full bridge (FB) converter by connecting the switch-nodes of the P-and

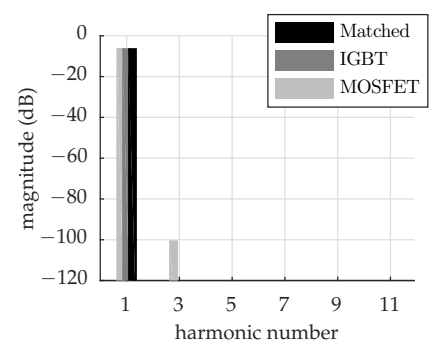

(a)

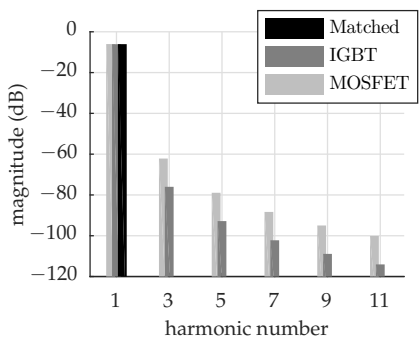

(b)
Fig. 7. Simulated output voltage spectra of a DB switching leg, normalized to the output voltage range, with ideal components for (a) constant bias current and (b) modulated bias current.

TABLE II

COMPONENT VALUES USED IN THE EXPERIMENTAL SETUP.

\begin{tabular}{lrll}
\hline Item & \multicolumn{2}{c}{ Value } & Description \\
\hline$L_{f}$ & 208 & $\mu \mathrm{H}$ & \\
$R_{L_{f}}$ & 50 & $\mathrm{~m} \Omega$ & \\
$C_{f}$ & 50 & $\mu \mathrm{F}$ & Vishay MKP1848650094Y5 \\
$C_{f_{\mathrm{DM}}}$ & 160 & $\mu \mathrm{F}$ & Vishay MKP1848716704Y5 \\
$L$ & 1.7 & $\mathrm{mH}$ & Air-cored inductor \\
$R$ & 2.5 & $\Omega$ & $1.5 \mathrm{~kW}$ wire wound resistor \\
\hline
\end{tabular}

$\mathrm{N}$-cell of each side of the full-bridge by means of a relay contact. Fig. 8 depicts the schematic diagram of the prototype converter. The switch nodes can be connected with the relays designated FB. The measured currents, required for feedback, are indicated in the figure.

The nominal component values of the experimental setup are summarized in Table II. The power supply voltage is chosen to be $100 \mathrm{~V}$, low enough to amplify the effects of the forward voltages of the switches and diodes. A resistorinductor load was used to represent the voice-coil actuator that is often used as load circuit for amplifiers used for short-stroke positioning and studio-quality audio systems. The switching frequency was $16 \mathrm{kHz}$ and the duty-ratios are updated twice per switching cycle. For the full-bridge configuration the blanking time $\left(T_{\mathrm{bt}}\right)$ was set to $2 \%$ of $T_{\mathrm{sw}}(1.25 \mu \mathrm{s})$. The offset current $i_{\text {th }}$ was set to $5.5 \mathrm{~A}$ for the DB configuration, which is enough to guarantee CCM.

Fig. 9 shows details of the experimental setup. The converter is based on an off-the-shelf Semikron IGBT stack with $6 \mathrm{mF}$ bus capacitance and a custom-designed gate-driver board based on Power Integrations SCALE2 drivers. It should be noted that this commercially available stack is by no means designed for generating low distortion waveforms.

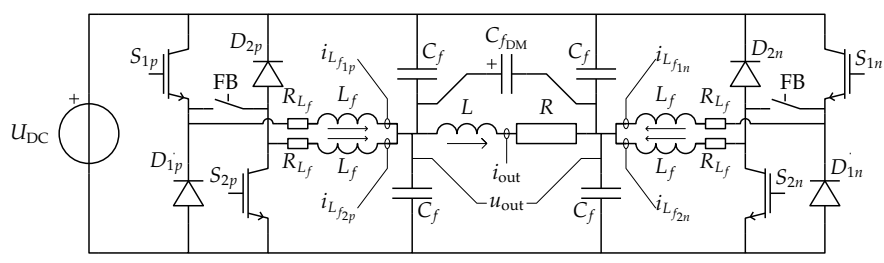

Fig. 8. Schematic overview of the reconfigurable full-bridge equivalent $\mathrm{DB}$ and conventional FB setup. 


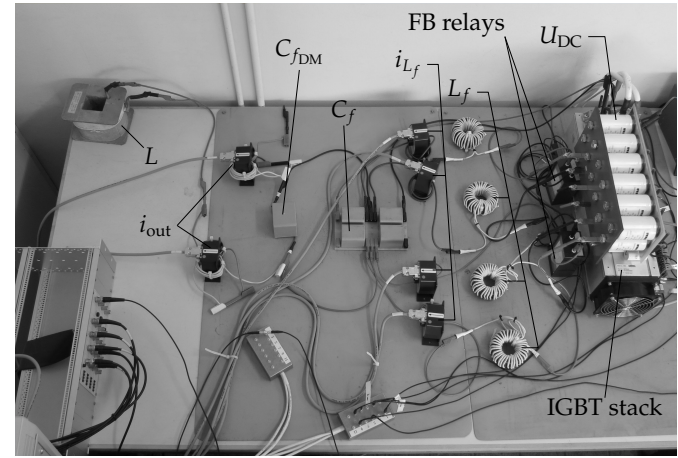

Fig. 9. Picture of the reconfigurable full-bridge equivalent $\mathrm{DB}$, and conventional FB setup.

The PWM generation and control are implemented on a dSPACE rapid prototyping system with an FPGA card on which the PWM modulators were implemented. The carrier phase shifts were set to bipolar switching.

The time-domain graphs in this section have been captured using an oscilloscope, and the frequency responses and spectra were measured using a Stanford Research Systems SR785 signal analyzer with typically better than $90 \mathrm{~dB}$ dynamic range. The output voltage spectra $\left(u_{\text {out }}\right)$ of the setup were directly measured using the differential input of the signal analyzer, through a custom $1 / 3$ attenuator made with $0.01 \%$ resistors having $1 \mathrm{ppm} / \mathrm{K}$ temperature coefficients. The output current was measured using an LEM IT200 Fluxgate sensor with a $5 \Omega$ burden resistor and 6 primary turns. All spectra were averaged 21 times using the RMS averaging function of the analyzer. This preserves the level of the (uncorrelated) noise while increasing resolution of the (correlated) spectral components.

To ensure CCM for the DB and good tracking of $i_{\text {bias }}$ for modulated bias, the bias currents were regulated with PI controllers that were assisted with simple first-order feedforward actions. Furthermore, to increase the PWM resolution, simple noise-shapers were implemented to dither the PWMs by adding the quantization errors to the next duty-ratios.

In all cases a sinusoidal reference was used for the output voltage with a frequency of $21 \mathrm{~Hz}$. The reference frequency was chosen extremely low to avoid distortion due to the regular sampled PWM. No feedback control was used to regulate the output quantity. The reference signal was directly fed into the modulator.

The THDs are evaluated over the first 38 harmonics and expressed in $\mathrm{dB}$, where $-80 \mathrm{~dB}$ corresponds to a THD of $0.01 \%$. The harmonic distortion (HD) of the power supply was calculated as

$$
\mathrm{HD}_{38}=\frac{\sqrt{\sum_{n=1}^{38}\left(U_{\mathrm{DC}}\left(n f_{o}\right)\right)^{2}}}{\left\langle U_{\mathrm{DC}}\right\rangle}
$$

where $\left\langle U_{\mathrm{DC}}\right\rangle$ represents the average supply voltage, in this case $100 \mathrm{~V}$, and the magnitude spectrum of the power supply voltage is indicated by $U_{\mathrm{DC}}(f)$.
From Fig. 10a it can be seen that the FB starts suffering from blanking-time-related distortion for amplitudes above $10 \mathrm{~V}$. For lower voltages the FB is operating in zero-voltageswitching mode, resulting in no blanking-time-related errors. The dashed line indicates the HD of the power supply, as given in (23), for the DB with constant bias current (cb). The power-supply plays a significant role in the distortion of the DB. For constant bias current the impact of the power supply is especially severe. For amplitudes above $40 \mathrm{~V}$ the THD of the DB with constant bias becomes approximately $6 \mathrm{~dB}$ lower than the corresponding HD of the power supply, which is expected when the THD is determined by power-supply intermodulation distortion [34].

Fig. 10b to Fig. 10d depict the voltage spectra for $50 \mathrm{~V}$ amplitude. Fig. 10b depicts the spectrum of the FB. The unwanted harmonic components are caused by the blanking time and the forward voltage of the IGBTs and diodes. The dominant spurious harmonic components for the DB in Fig. 10c originate from the power supply, as pointed out before. When comparing the measurement results of Fig. 10d with the simulation results of the IGBT case in Fig. 7b it can be seen that the measured spurious spectral components due to modulated bias are significantly higher than the simulated case. However, the amplitude decay of the first three unwanted harmonics of the measurement is similar to the simulation results. As such, the first three odd harmonics in Fig. 10d can be attributed to the use of modulated bias. Part of the amplitude difference can be due to parameter mismatch between the simulation model and the actual set-up. However, voltage commutation and reverse recovery that were neglected in the simulation study, which adds to distortion too. Also higher order effects like temperature dependencies, and the exponential nature of the transistors and diodes contribute to the difference. The simulation study does not include these effects because they would distract from the scope of this paper, which is to show that the DB converter does not suffer from zero crossing distortion due to blanking-time and forward voltages of the semiconductors, and that its cycle average behavior is linear when $R_{\mathrm{on}}$ and $R_{f}$ are matched. However, the performance of the DB is still impressive when considering that the setup is based on high-power $1200 \mathrm{~V}$ IGBTs operated at only $100 \mathrm{~V}$ and that the output is modulated open-loop without feedback.

The corresponding time waveforms, with $80 \%$ output voltage modulation, for the FB and DB with constant and modulated bias current are shown in Figures 11. The distortion of the output voltage and current waveforms is barely visible for the FB case, and not visible at all for the DB cases, as can be expected from the measured THDs shown in Fig. 10. Modulated bias will result in significantly lower losses compared to constant bias, in this particular case approximately $35 \%$ less. When comparing the measured THDs and corresponding spectra of the DB to the FB in Fig. 10 it can be seen that the DB with constant bias current performs over 100 times better than the conventional hard-switching FB. Even with modulated bias the THD of the DB is still 30 times better than the hardswitching FB. The performance for a zero-voltage-switching FB approaches the performance of the DB with modulated 


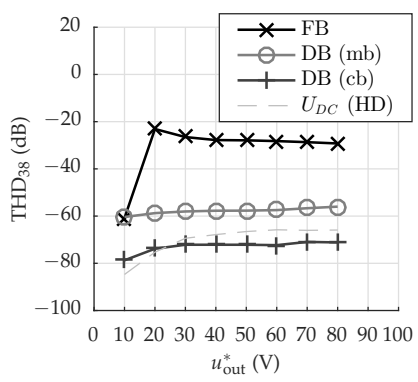

(a)

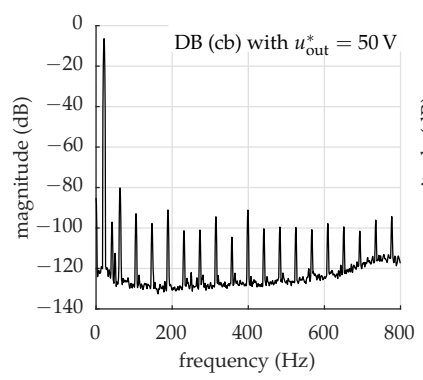

(c)

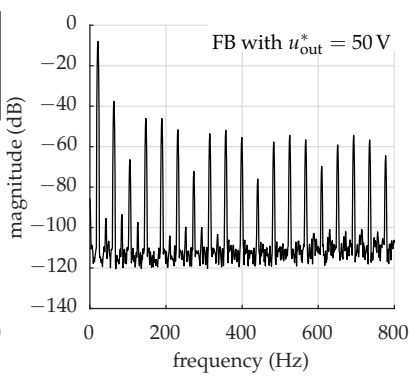

(b)

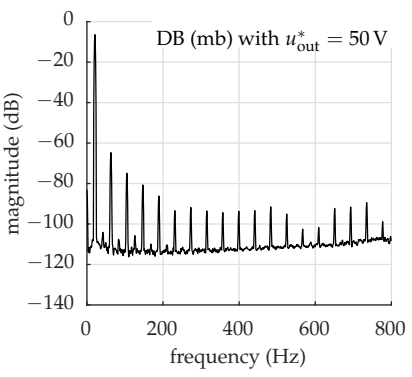

(d)
Fig. 10. Measured results under open-loop conditions, (a) THD of different converters as function of output voltage setpoint, and voltage magnitude spectra for $50 \mathrm{~V}$ setpoint of (b) FB, (c) DB with constant bias, and (d) DB with modulated bias.

bias. For constant bias current the THD of the DB remains significantly better than the zero-voltage-switching FB.

\section{CONCLUSIONS}

This paper focused on the open-loop modulation of the dual buck converter (DB). A decoupled modulation scheme is proposed that allows separate control of the output quantity and the bias current which is required to maintain continuous conduction mode (CCM).

The impact of component parameters on the output quality is investigated. It is shown that when both legs of the DB are operated in CCM the cycle average behavior becomes linear when the on-resistances of the semiconductor switches and diodes are matched. Moreover, when the inductors of the DB legs are chosen equal there is no cross-coupling between the output quantity and the bias current, which allows decoupled control of the bias needed to guarantee CCM, and the output quantity. The bias current can be chosen constant for the best output quality or can be modulated to reduce losses at the cost of higher distortion due to component variation of the filter inductors.

Measurements were done on an industrial IGBT stack based prototype converter that can be switched between the conventional FB and its DB equivalent. The measurements confirm the superior output quality of the DB converter and show that the THD of the DB is up to 2 orders of magnitude less than the conventional FB. The measurements prove that the DB can be used as an open-loop switched mode amplifier for high precision applications like audio, MRI, and shortstroke servo systems.

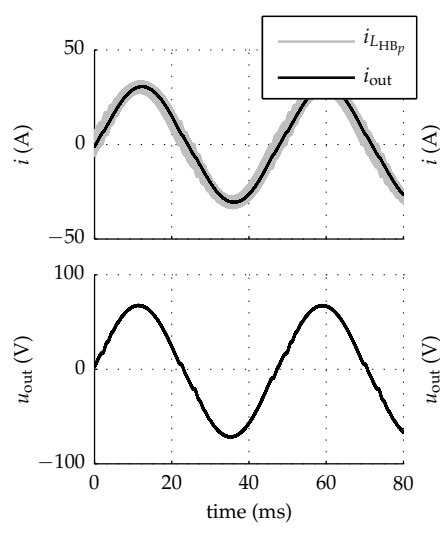

(a)

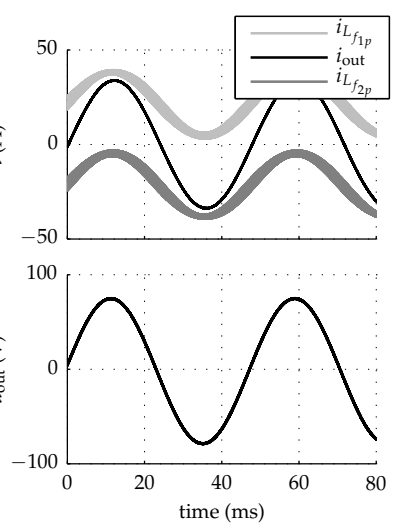

(b)
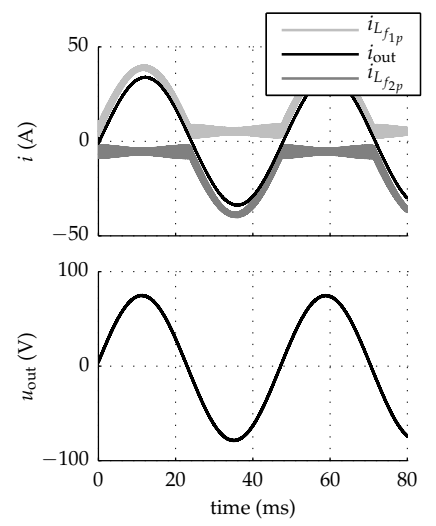

(c)

Fig. 11. Measured waveforms for sinusoidal reference with $80 \mathrm{~V}$ amplitude for (a) the FV, (b) the DB with constant bias, and (c) the DB with modulated bias (corresponding to the spectra shown in Fig. 10).

\section{REFERENCES}

[1] J. M. Schellekens, J. L. Duarte, H. Huisman, and M. A. M. Hendrix, "Elimination of zero-crossing distortion for high-precision amplifiers," in Proceedings of the $37^{\text {th }}$ Annual Conference of the IEEE Industrial Electronics Society (IECON), pp. 3370-3375, 2011.

[2] B. Bidoggia, R. Maheshwari, R. Nielsen, S. Munk-Nielsen, and F. Blaabjerg, "Steady-state analysis of dead-time effect on bidirectional buck converters," in Proceedings of the $38^{\text {th }}$ Annual Conference of the IEEE Industrial Electronics Society (IECON), pp. 792-797, 2012.

[3] M. L. A. Caris, H. Huisman, J. M. Schellekens, and J. L. Duarte, "Generalized harmonic elimination method for interleaved power amplifiers," in Proceedings of the $38^{\text {th }}$ annual Conference of the IEEE Industrial Electronics Society (IECON), pp. 4961-4966, 2012.

[4] M. L. A. Caris, "Exploring the powerDAC : an asymmetric multilevel approach for high-precision power amplification," Ph.D. dissertation, Eindhoven University of Technology, 2016.

[5] E. Lemmen, J. van Duivenbode, and J. L. Duarte, "Load-currentcorrected capacitor voltage control in eight-level DC-AC converter using extended commutation cells," IEEE Transactions on Power Electronics, vol. 31, no. 10, pp. 7286-7296, Oct. 2016.

[6] Y.-K. Lin and Y.-S. Lai, "Dead-time elimination of PWM-controlled inverter/converter without separate power sources for current polarity detection circuit," IEEE Transactions on Industrial Electronics, vol. 56, no. 6, pp. 2121-2127, Jun. 2009.

[7] C. Attaianese, V. Nardi, and G. Tomasso, "A novel SVM strategy for VSI dead-time-effect reduction," IEEE Transactions on Industry Applications, vol. 41, no. 6, pp. 1667-1674, nov.-dec. 2005.

[8] N. Urasaki, T. Senjyu, K. Uezato, and T. Funabashi, "Adaptive deadtime compensation strategy for permanent magnet synchronous motor drive," IEEE Transactions on Energy Conversion, vol. 22, no. 2, pp. 271-280, Jun. 2007. 
[9] D. Leggate and R. J. Kerkman, "Pulse-based dead-time compensator for PWM voltage inverters," IEEE Transactions on Industrial Electronics, vol. 44, no. 2, pp. 191-197, Apr. 1997.

[10] G. Pellegrino, R. I. Bojoi, P. Guglielmi, and F. Cupertino, "Accurate inverter error compensation and related self-commissioning scheme in sensorless induction motor drives," IEEE Transactions on Industry Applications, vol. 46, no. 5, pp. 1970-1978, 2010.

[11] J. M. Schellekens, R. A. M. Bierbooms, and J. L. Duarte, "Deadtime compensation for PWM amplifiers using simple feed-forward techniques," in Proceedings of the XIX ${ }^{\text {th }}$ International Conference on Electrical Machines (ICEM), pp. 1-6, 2010.

[12] T. Mannen and H. Fujita, "Dead time compensation method based on current ripple estimation," in Proceedings of the IEEE Energy Conversion Congress and Exposition (ECCE), pp. 775-782, 2013.

[13] K. M. Cho, W. S. Oh, Y. T. Kim, and H. J. Kim, "A new switching strategy for pulse width modulation (PWM) power converters," IEEE Transactions on Industrial Electronics, vol. 54, no. 1, pp. 330-337, 2007.

[14] M. Berkhout, "A class D output stage with zero dead time," in Digest of technical papers of the IEEE International Solid-State Circuits Conference (ISSCC), pp. 134-135, 2003

[15] M. Rose, J. Krupar, H. Guldner, and E. Brenner, "Effects of varying load conditions on adaptive gate control methods," in Proc. 2011-14th European Conf. Power Electronics and Applications (EPE 2011), pp. $1-7,2011$

[16] V. Yousefzadeh and D. Maksimovic, "Sensorless optimization of dead times in DC-DC converters with synchronous rectifiers," IEEE Transactions on Power Electronics, vol. 21, no. 4, pp. 994-1002, 2006.

[17] Y. Lobsiger and J. W. Kolar, "Closed-loop $\mathrm{d} i / \mathrm{d} t$ and $\mathrm{d} v / \mathrm{d} t$ control and dead time minimization of IGBTs in bridge leg configuration," in Proceedings of the $14^{\text {th }}$ IEEE workshop on Control and Modeling for Power Electronics (COMPEL), pp. 1-7, Jun. 2013.

[18] D. M. Divan and G. Skibinski, "Zero-switching-loss inverters for high-power applications," IEEE Transactions on Industry Applications, vol. 25, no. 4, pp. 634-643, 1989.

[19] J. Cho, D. Hu, and G. Cho, "Three phase sine wave voltage source inverter using the soft switched resonant poles," in Proceedings of the $15^{\text {th }}$ Annual Conference of the IEEE Industrial Electronics Society (IECON), vol. 1, pp. 48-53, Nov. 1989.

[20] R. W. De Doncker and J. P. Lyons, "The auxiliary resonant commutated pole converter," in Conference record of the IEEE Industry Applications Society (IAS) annual meeting, pp. 1228-1235, 1990.

[21] N. R. Zargari, P. D. Ziogas, and G. Joos, "A two-switch highperformance current regulated DC/AC converter module," IEEE Transactions on Industry Applications, vol. 31, no. 3, pp. 583-589, 1995.

[22] G. Stanley and K. M. Bradshaw, "Precision DC-to-AC power conversion by optimization of the output current waveform - the half bridge revisited," IEEE Transactions on Power Electronics, vol. 14, no. 2, pp. 372-380, Mar. 1999.

[23] J. Liu and Y. Yan, "A novel hysteresis current controlled dual buck half bridge inverter," in Proceedings of the $34^{\text {th }}$ Annual IEEE Power Electronics Specialist Conference (PESC), vol. 4, pp. 1615-1620, 2003.

[24] C. Zhu, F. Zhang, and Y. Yan, "A novel split phase dual buck half bridge inverter," in Proceedings of the $20^{\text {th }}$ Annual IEEE Applied Power Electronics Conference and Exposition (APEC), vol. 2, pp. 845-849, 2005.

[25] J. M. Schellekens, "A class of robust switched-mode power amplifiers with highly linear transfer characteristics, on the elimination of zerocrossing distortion in switching converters," Ph.D. dissertation, Eindhoven University of Technology, 2014.

[26] E. Lemmen, J. M. Schellekens, C. G. E. Wijnands, and J. L. Duarte, "Advances in high precision amplifiers - the extra L opposed current converter," IEEE Transactions on Power Electronics, vol. 30, no. 10, pp. 5691-5700, Oct. 2015.

[27] J. M. Schellekens, M. L. A. Caris, J. L. Duarte, H. Huisman, M. A. M. Hendrix, and E. A. Lomonova, "High precision switched-mode amplifier with an auxiliary bias circuit," in Proceedings of the $15^{\text {th }}$ European Conference on Power Electronics and Applications (EPE), pp. 1-10, 2013.

[28] R. W. Erickson and R. D. Middlebrook, "Origins of harmonic distortion in switching amplifiers," in Proceedings of the $4^{\text {th }}$ annual International Power Conversion Conference, pp. 567-582, 1982.

[29] R. W. Erickson, "Large signals in switching converters," Ph.D. dissertation, California Institute of Technology, 1983.

[30] K. Nielsen, "Audio power amplifier techniques with energy efficient power conversion, volume I," Ph.D. dissertation, Technical University of Denmark (DTU), 1998.
[31] M. Mauerer, A. Tüysüz, and J. W. Kolar, "Distortion analysis of low-THD/high-bandwidth GaN/SiC class-D amplifier power stages," in Proceedings of the IEEE Energy Conversion Congress and Exposition (ECCE), 2015.

[32] R. Middlebrook and S. Cuk, "A general unified approach to modelling switching-converter power stages," in Proceedings of the IEEE Power Electronics Specialists Conference (PESC), vol. 1, pp. 18-34, 1976.

[33] D. G. Holmes and T. A. Lipo, Pulse Width Modulation for Power Converters: Principles and Practice (IEEE Press Series on Power Engineering). Wiley-IEEE Press, 2003.

[34] M. Berkhout, "An integrated 200-W class-D audio amplifier," IEEE journal of solid-state circuits, vol. 38, no. 7, pp. 1198-1206, Jul. 2003.

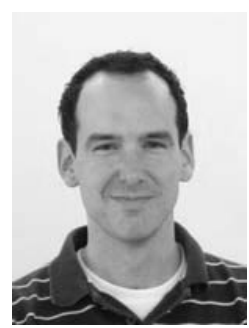

Jan M. Schellekens was born in Goirle, the Netherlands on January 9, 1978. He received the B.E. degree in electrical engineering from Fontys University of Applied Sciences, Eindhoven, The Netherlands, in 2001, and the M.Sc. degree in electrical engineering, specializing in power electronics from Eindhoven University of Technology, Eindhoven, in 2007. After gaining several years of experience in industry he joined the Electromechanics and Power Electronics Group of the Eindhoven University of Technology, where he obtained the Ph.D. degree in 2014. Currently, he is working as a system architect at AME, a supplier of high-tech electronics in Eindhoven, and is part time active as assistant professor at the Eindhoven University of Technology. His research interests include power electronics for high precision applications, and control design.

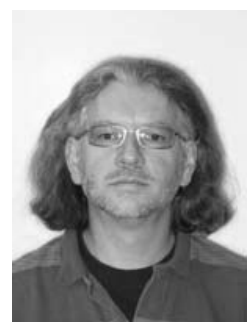

Henk Huisman (M'93-SM'17) was born in Gouda, The Netherlands, in 1958. He received the MSc. degree in 1984, and the PhD. degree in 1992, both from Delft University of Technology, The Netherlands. After graduating, he has been employed as a research scientist at Delft University in the Laboratory for Power Electronics and also in the Systems and Control Group. Later he joined CCM (Nuenen, The Netherlands) where he worked on high speed drive systems for flywheel applications and energy management. From 2006 to 2015 he was a staff member of Philips Electronics, Eindhoven, The Netherlands, working on power electronics, mostly for medical applications. In 2015 and 2016 he was employed by ATB Technologies Netherlands B.V., performing research on and design of electronic power converters for drive applications. In 2010 he was also appointed as a part-time assistant professor at Eindhoven University of Technology, where he has been working on the analysis and design of high-precision power amplifiers and resonant power converters. In 2016 he was appointed associate professor in Power Electronics, with a special focus on Electric Drives. His teaching tasks comprise courses on power electronics, on wireless energy transfer and on electric drives.

$\mathrm{He}$ has authored papers on multiphase series-resonant power converters, flywheel applications, and various other topics. His primary professional interests lie in power electronics, application of chaos theory in system development, simulation, and control design. 


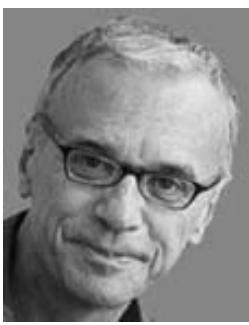

Jorge L. Duarte (M'99) received the M.Sc. degree from the Federal University of Rio de Janeiro, Rio de Janeiro, Brazil, in 1980, and the Dr.-Ing. degree from the Institute National Polytechnique de Lorraine, Nancy, France, in 1985. In 1989, he was appointed a Research Engineer at Philips Lighting Central Development Laboratory. Since 1990, he has been a Member of the academic staff with the Electromechanics and Power Electronics Group, Eindhoven University of Technology, Eindhoven, The Netherlands. Since October 2000, he has been a Consultant Engineer on a regular basis at high-tech industries around Eindhoven. In 2008, he was an Invited Lecturer with Zhejiang University, Hangzhou, China. His teaching and research interests include modeling, simulation, and design optimization of power electronic systems.

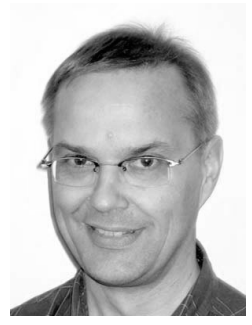

Marcel A. M. Hendrix (M'98) received the M.Sc. degree in electronic circuit design from the Eindhoven University of Technology (TU Eindhoven), Eindhoven, The Netherlands, in 1981. He is currently a Senior Electronics Architect at Philips Innovation Services, SC, Eindhoven. In 1983 he joined Philips Lighting, Eindhoven, where he started to work in the Pre-Development Laboratory, Business Group Lighting Electronics and Gear (BGLE\&G). Since that time he has been involved in the design and specification of switched power supplies for solar inverters, low and high pressure gasdischarge lamps, and LEDs. In July 1998, he was appointed a parttime Professor (UHD) with the Electromechanics and Power Electronics Group, TU Eindhoven, where he teaches a design-oriented course in power electronics below $4 \mathrm{~kW}$. His professional interests are with system simulation, device characterization and modeling, real-time programming, and embedded digital control.

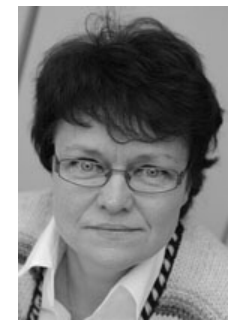

Elena A. Lomonova (M'04-SM'07) Full Professor, Chair of Electromechanics and Power Electronics Group Prof. dr. Elena Lomonova studied Electromechanical and Control Systems at Moscow Aviation Institute - (State University of Aerospace Technology), Russia. After graduating (cum laude), she started her industrial carrier at the Research and Development Company "Astrophysics", Moscow, Russia (1982-1987). Afterwards she moved to the Electromechanical and Control Systems Department at State University of Aerospace Technology (MAl), and was active in research, education and industrial projects (1987-1997). She gained her PhD (cum laude, 1993) on researching of powertrain and control systems for autonomous vehicles with multi-level power supply subsystems for on-board loads and laser equipment. Since 1998 she worked for the Delft University of Technology before joining Eindhoven University of Technology in 2000. In March 2009 she was appointed as a full-time professor. Her chair focuses on fundamental and applied research on enabling energy conversion theory, methods and technologies for high-precision, automotive and medical systems. Her research activities span various facets of advanced mechatronics, electromechanics and electromagnetics including rotary electrical machines and drives, linear and planar actuation systems. She is an author and co-author of more than 450 scientific publications and more than 10 patents. 